\title{
Editorial
}

\section{Half-Full or Half-Empty}

\section{Thomas C. Hulsey MSPH, ScD}

Journal of Perinatology (2003) 23, 609. doi:10.1038/sj.jp.7211016

The article by Dr. Strobino et al. ${ }^{1}$ in this issue of the Journal provides insight into the intricate nature of relationships between components of the perinatal health-care system. Their investigation reveals the dynamic environment of the relationships between hospitals and between providers and how these relationships change in relation to the changes in number of hospitals and whether or not there were formal state guidelines to designate hospital level of care.

State guidelines designating hospital level of care occurred in $63 \%$ of communities, and in $79 \%$ of these, the guidelines were formalized. At first glance, this seems impressive but the alternative message is that approximately $50 \%$ of the communities surveyed (79\% of 63\%) have no formal state guidelines to designate hospital level of care. In addition, over one-third (37\%) of the communities surveyed had no state guidelines of any sort to designate hospital level of care!

Linkages between nontertiary and tertiary hospitals were reported in $65 \%$ of communities and among these, $65 \%$ of these linkages were formal. Again this seems impressive but the alternative interpretation is that there were no linkages of any sort between hospitals in 35\% of communities. There were no formal linkages between tertiary and nontertiary hospitals in $58 \%$ of the communities surveyed.

Approximately $74 \%$ of communities reported that there were hospital linkages that involved agreements about pediatric or NICU consultation or about emergency transport of medically high-risk newborns. In other words, in $26 \%$ of communities where there are hospitals providing perinatal care, there are no such agreements in place.

In states where no guidelines designated hospital level of care, $55 \%$ of maternity and newborn hospitals had linkages, but this increased to $87 \%$ in states where guidelines existed. This underscores the importance of having state oversight with respect to hospital level designation. Hospitals in communities with formal state guidelines were 1.6 times more likely to have linkages with other hospitals as compared to hospitals in states without formal guidelines.
These findings underscore the importance of examining not only the individual components of a perinatal health-care system, but also the nature of interaction between them and the importance of formalized state guidelines. This paper comes at a time when the nature of regionalized perinatal care is undergoing significant metamorphosis. Perhaps one of the most surprising findings in this study was that a substantial percentage of communities surveyed indicated a complete lack of state oversight into how their perinatal health-care systems were evolving.

State guidelines appeared to be integral in the existence of agreements between providers and linkages between hospitals. In a system that builds its foundation on the concept of 'risk appropriate care' and the recommendations of the guiding documents of TIOP I and TIOP II, the importance of state involvement to assist the system of hospitals and providers towards that goal should not be diminished. ${ }^{2,3}$ Indeed, a system composed of such complex inter-relationships between independent, yet linked, components needs external nonpartisan oversight to steer the whole dynamic process. The existence of these needed relationships between components is absent in those states without such guidelines. Dr. Strobino et al. have provided a very important insight into communities around the country. Thankfully, the majority seems to have the structure in place to support the linkages and relationships needed between the components in the system of perinatal care. At the same time, there are a surprising number of communities where the structure of the perinatal health-care system evolves unregulated. Although state guidance does not necessarily guarantee the optimal system of care, left to the partisan nature of the individual components, such systems might be less likely to evolve naturally into optimal systems of care.

\section{References}

1. Strobino DM, Silver GB, Allston AA, Grayson HA. Local health department perspectives on linkages among birthing hospitals. J Perinatol, this issue.

2. Committee on Perinatal Health. Toward improving the outcome of pregnancy: recommendations for the regional development of maternal and perinatal health services (TIOP I). March of Dimes Birth Defects Foundation, 1976

3. Committee on Perinatal Health. Toward improving the outcome of pregnancy: the 90s and beyond (TIOP II). March of Dimes Birth Defects Foundation, 1993. 